\title{
Sufficient Conditions for Topological Invariance of 2D Images under Rigid Transformations ${ }^{\star}$
}

\author{
Phuc Ngo ${ }^{1}$, Yukiko Kenmochi ${ }^{1}$, Nicolas Passat ${ }^{2}$, and Hugues Talbot ${ }^{1}$ \\ 1 Université Paris-Est, LIGM, UPEMLV-ESIEE-CNRS, France \\ ${ }^{2}$ Université de Reims, CReSTIC, EA 3804, France
}

\begin{abstract}
In $\mathbb{R}^{2}$, rigid transformations are topology-preserving operations. However, this property is generally no longer true when considering digital images instead of continuous ones, due to digitization effects. In this article, we investigate this issue by studying discrete rigid transformations (DRTs) on $\mathbb{Z}^{2}$. More precisely, we define conditions under which digital images preserve their topological properties under any arbitrary DRTs. Based on the recently introduced notion of DRT graph and the classical notion of simple point, we first identify a family of local patterns that authorize topological invariance under DRTs. These patterns are then involved in a local analysis process that guarantees topological invariance of whole digital images in linear time.
\end{abstract}

Keywords: 2D digital image, discrete rigid transformation, topology, simple point, DRT graph, Eulerian model.

\section{Introduction}

In $2 \mathrm{D}$, rigid transformations (i.e., rotations composed with translations) are involved in numerous image processing/analysis tasks, e.g., registration [1] or tracking [2. In such applications, the images are generally digital, and can then be considered as functions $I: S \rightarrow F$ from a finite subset $S \subset \mathbb{Z}^{2}$ to a value space $F$. While rigid transformations are topology-preserving operations in $\mathbb{R}^{2}$, this property is generally lost in $\mathbb{Z}^{2}$, due to the discontinuities induced by the mandatory digitization from $\mathbb{R}$ to $\mathbb{Z}$. In particular, discrete rigid transformations (DRTs) - that include discrete rotations 3 4 5 6] - are not guaranteed to preserve the homotopy type of digital images, as exemplified in Fig. 1.

In this article, we study this specific issue. More precisely, we investigate some conditions under which digital images preserve their topological properties under any arbitrary DRTs, by considering the Eulerian (i.e., backwards) transformation model. To reach this goal, we consider $(i)$ the notion of DRT graph, recently introduced by the authors in 78 , that defines a combinatorial model of all the rigid transformations of a digital image, and $(i i)$ the classical notion of simple point 910, that provides sufficient conditions to guarantee the preservation of homotopy type.

* The research leading to these results has received funding from the French Agence Nationale de la Recherche (Grant Agreement ANR-2010-BLAN-0205 03).

R. Gonzalez-Diaz, M.-J. Jimenez, B. Medrano (Eds.): DGCI 2013, LNCS 7749, pp. 155-168, 2013.

(C) Springer-Verlag Berlin Heidelberg 2013 

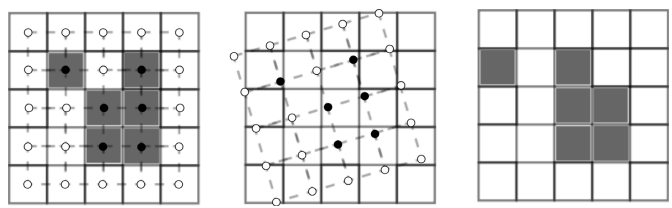

Fig. 1. Left: a binary digital image and the grid modeling its discrete structure. Middle: a rigid transformation applied on this grid. Right: the resulting transformed image, with a homotopy different from the initial one (the black pixels, in the 8-adjacency, have been split).

By combining these two notions, we first propose a way to determine transformed images which have the same homotopy type as the initial one, by scanning the whole DRT graph associated to this image. Then, we show that this global approach, which presents a polynomial complexity, can be simplified into a local approach, based on a spatial decomposition of the image into covering samples. In order to do so, we identify a family of local patterns that authorize topological invariance under DRTs. These patterns can then be involved in a procedure based on look-up tables (LUT) that guarantee topological invariance of a whole digital image in linear time.

The article is organised as follows. Sec. 2 presents background notions related to rigid transformations and digital topology. Sec. 3 describes the topological issues induced by DRTs. Sec. 4 explains how DRT graphs and simple points can be combined to evaluate topological invariance under DRTs, leading to an algorithm detailed in Sec. 5] Experiments are proposed in Sec. 6] while Sec. 7 concludes the article.

\section{Background Notions}

\section{1 (Discrete) Rigid Transformations}

In $\mathbb{R}^{2}$, a rigid transformation (i.e., a transformation composed of a translation and a rotation) is expressed as a bijection $\mathcal{T}: \mathbb{R}^{2} \rightarrow \mathbb{R}^{2}$ defined, for any $\boldsymbol{x}=$ $(x, y) \in \mathbb{R}^{2}$ by

$$
\mathcal{T}(\boldsymbol{x})=\left(\begin{array}{rr}
\cos \theta & -\sin \theta \\
\sin \theta & \cos \theta
\end{array}\right)\left(\begin{array}{l}
x \\
y
\end{array}\right)+\left(\begin{array}{l}
a \\
b
\end{array}\right) \quad \text { with } a, b \in \mathbb{R} \text { and } \theta \in[0,2 \pi[
$$

Such a transformation (also noted $\mathcal{T}_{a b \theta}$ ) is unambiguously modeled by the triplet of parameters $(a, b, \theta)$. It is not possible to apply directly $\mathcal{T}$ on a digital image $I: S \rightarrow F$, since there is no guarantee that $\mathcal{T}(\boldsymbol{x}) \in \mathbb{Z}^{2}$, for any $\boldsymbol{x} \in S \subset \mathbb{Z}^{2}$. The handling of discrete rigid transformations (DRTs) then requires the definition of a function $T: \mathbb{Z}^{2} \rightarrow \mathbb{Z}^{2}$, which is the "discrete analogue" of $\mathcal{T}$. Considering the standard rounding function $D: \mathbb{R}^{2} \rightarrow \mathbb{Z}^{2}$, this can be conveniently performed by setting $T=D \circ \mathcal{T}$, as illustrated on the diagram below. 


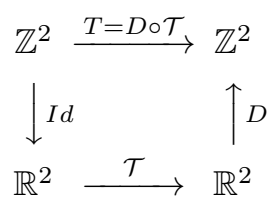

The function $T: \mathbb{Z}^{2} \rightarrow \mathbb{Z}^{2}$ is then explicitly defined, for any $\boldsymbol{p}=(p, q) \in \mathbb{Z}^{2}$, by

$$
T(\boldsymbol{p})=D \circ \mathcal{T}(\boldsymbol{p})=\left(\begin{array}{l}
{[p \cos \theta-q \sin \theta+a]} \\
{[p \sin \theta+q \cos \theta+b]}
\end{array}\right)
$$

In general, this function is not bijective. However, by setting $T^{-1}=D \circ \mathcal{T}^{-1}$ : $\mathbb{Z}^{2} \rightarrow \mathbb{Z}^{2}$, we can define the transformed digital image $I \circ T^{-1}: \mathbb{Z}^{2} \rightarrow F$ with respect to $T$. Note that $T^{-1}$ is not the inverse function of $T$ in general.

\subsection{Digital Topology}

Several frameworks are available to model the topological structure of a digital image. In $\mathbb{Z}^{2}$, most of these frameworks (see, e.g., [11]12]) can be conveniently unified within the frequently used -and also simple- framework of digital topology [9]. In this framework, the topological notions derive from a graph structure induced by two adjacency relations, namely the 4- and 8-adjacencies, which are defined for any two points $\boldsymbol{p}, \boldsymbol{q} \in \mathbb{Z}^{2}$ such that $\boldsymbol{p}$ and $\boldsymbol{q}$ are 4-adjacent (resp. 8-adjacent) if $\|\boldsymbol{p}-\boldsymbol{q}\|_{1} \leq 1$ (resp. if $\|\boldsymbol{p}-\boldsymbol{q}\|_{\infty} \leq 1$ ). It is well known that, to deal with topological paradoxes related to the digital version of the Jordan theorem, we generally use in one binary digital image a pair of different adjacency relations, and denote as $(\alpha, \beta)$ where $\alpha$ and $\beta$ are adjacency relations for foreground (black) and background (white) pixels respectively. In $2 \mathrm{D}$, we consider in particular $(\alpha, \beta)=(4,8)$ or $(8,4)$.

In the graph-based framework of digital topology, the concept of simple point 910 (see Fig. 2) relies on the local notion of adjacency and on the induced global notion of connectedness. The simple points provide a way to characterise the preservation of topological properties in a (binary) image during its transformation. Practically, a pixel $\boldsymbol{x} \in S$ of an image $I: S \rightarrow F$ is simple if its binary value can be switched without modifying the topological properties of I. In particular, the simplicity of a pixel can be tested, in constant time, by only studying its $3 \times 3$ neighbourhood [9]. We will say that two images $I$ and $I^{\prime}$ are simple-equivalent 13 if $I^{\prime}$ is obtained from $I$ by iteratively modifying (successive) simple points. Thus $I$ and $I^{\prime}$ present the same homotopy type.

\section{Discrete Rigid Transformations: Topological Issues}

A (continuous) rigid transformation $\mathcal{T}$ establishes a bijection from $\mathbb{R}^{2}$ to itself. By opposition, due to the digitisation process $D$ (see Eq. (2)), a discrete rigid transformation $T$ is, most of the time, not a bijection from $\mathbb{Z}^{2}$ to itself.

It is plain that for any three distinct pixels $\boldsymbol{x}_{\mathbf{1}}, \boldsymbol{x}_{\mathbf{2}}, \boldsymbol{x}_{\boldsymbol{3}} \in \mathbb{Z}^{2}$, we have $\max _{i, j \in\{1,2,3\}}\left\{\left\|\boldsymbol{x}_{\boldsymbol{i}}-\boldsymbol{x}_{\boldsymbol{j}}\right\|_{2}\right\} \geq \sqrt{2}$. This leads to the following definition that 


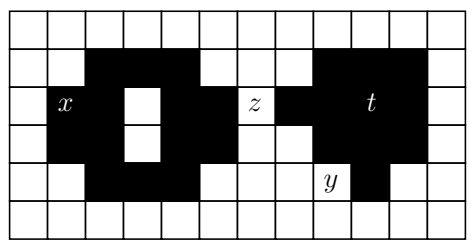

Fig. 2. Examples of simple points $(\boldsymbol{x}, \boldsymbol{y})$ and non-simple points $(\boldsymbol{z}, \boldsymbol{t})$. Modifying the value of $\boldsymbol{z}$ would merge two black connected components, while modifying the value of $\boldsymbol{t}$ would create a white connected component. In both cases, the homotopy type of the image would be modified.
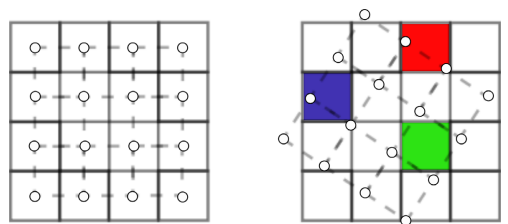

Fig. 3. Left: a digital image support and the grid modeling its discrete structure. Right: examples of a null pixel (in green), a single pixel (in blue) and a double pixel (in red) with respect to a discrete rigid transformation.

enables to characterise the status of a pixel; there are only three possibilities, as illustrated in Fig. 3 .

Definition 1. For a pixel $\boldsymbol{x} \in \mathbb{Z}^{2}$ and a given DRT $T$, let $M(\boldsymbol{x})=\left\{\boldsymbol{y} \in \mathbb{Z}^{2} \mid\right.$ $T(\boldsymbol{y})=\boldsymbol{x}\}$.

- If $|M(\boldsymbol{x})|=0$, we say that $\boldsymbol{x}$ is a null pixel.

- If $|M(\boldsymbol{x})|=1$, we say that $\boldsymbol{x}$ is a single pixel.

- If $|M(\boldsymbol{x})|=2$, we say that $\boldsymbol{x}$ is a double pixel.

Similar notions for the case of discrete rotations can be found in [56].

In particular, a discrete rigid transformation $T$ behaves like a bijection for single pixels. However the possible existence of null (resp. double) pixels may forbid $T$ to be a surjection (resp. an injection). Null and double pixels thus raise topological issues in both Lagrangian and Eulerian transformation models (see Sec. 3.1). In addition to these "cardinality-based" issues, supplementary topological problems are induced by the alteration of adjacency relations between pixels (see Sec. 3.2).

\subsection{Transformation Models}

Two standard transformation models can be considered: the Lagrangian (or forwards) and the Eulerian (or backwards) models. The Lagrangian model consists of observing $\mathcal{T}(\boldsymbol{x})$ for every pixel $\boldsymbol{x}$ in the initial space, while the Eulerian model consists of observing $\mathcal{T}^{-1}(\boldsymbol{y})$ for every pixel $\boldsymbol{y}$ in the transformed space. These models are equivalent in $\mathbb{R}^{2}$, since $\mathcal{T}$ is bijective. In $\mathbb{Z}^{2}$, they are however generally distinct, since $T$ and $T^{-1}$ may not be inverse functions. 


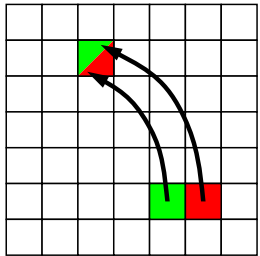

(a) Lagrangian model
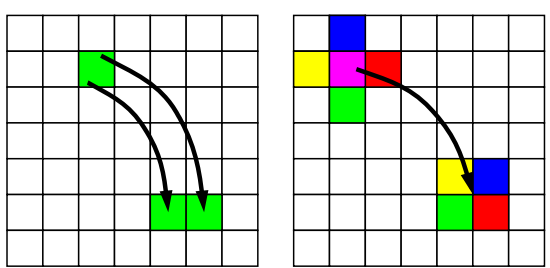

(b) Eulerian model

Fig. 4. The interpretations of double pixels (left figures) and null pixels (right figures) in the context of discrete rigid transformations for (a) the Lagrangian and (b) the Eulerian models

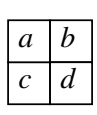

(a)

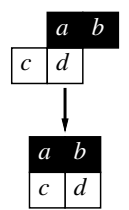

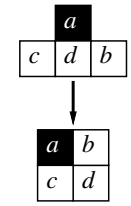

(c)
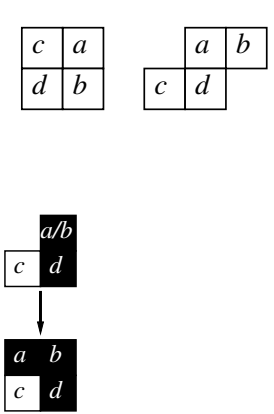

(b)
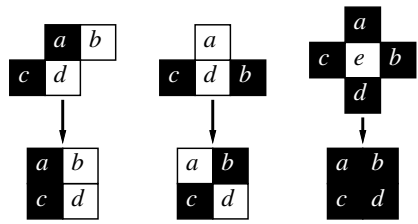

(d)

Fig. 5. (a) A $2 \times 2$ pixel sample with values $a, b, c, d$. (b) Local pixel configurations (up to rotations and symmetries) leading to the sample (a) when applying a discrete rigid transformation. (c) Examples of transformations in which the sample preserves the topology of local pixel configurations. (d) Examples of transformations in which the sample provokes a topological alteration.

Depending on each model, null and double pixels lead to different interpretations. In the Lagrangian model (see Fig. 4(a)), a double pixel in the transformed space may receive two different pixel values, and a null pixel receive no pixel value. While this may be conveniently handled in the case of binary images (which can be considered in a set-based paradigm), it can lead to correctness and completeness issues in the case of multivalued images. In the Eulerian model (see Fig. 4(b)), a double pixel of the initial space may transfer its value to two pixels of the transformed space, while the value of a null pixel will be lost.

In this preliminary work, we consider the Eulerian model which enables us to focus on the topological issues raised by the alteration of adjacency relations (see Sec. 3.2), and as the Lagrangian model is fraught with additional difficulties (see Sec. 7). For the sake of readability, our study is carried out in the context of binary images, but the introduced methodology remains valid in the case of multivalued images (see Sec. 7). 


\subsection{Adjacency Alterations}

In order to illustrate the topological issues raised by the alterations of adjacency relations during discrete rigid transformations, let us consider a $2 \times 2$ pixel sample of the transformed space (see Fig. 5(a)). Such a sample is composed of pixels of values $a, b, c$ and $d$, and all the possible local pixel configurations of the initial space from which the sample is generated (see Fig. 5(b)). Despite local adjacency alterations between pixels, the global topology of the sample may sometimes be preserved (see Fig. 5(c)). Unfortunately, such local alterations may also lead to topological alterations in the sample (see Fig. 5(d)), and further in the whole image possibly. In the next section, we propose an algorithm enabling the detection of potential topological changes during a DRT. On the contrary, this algorithm can be used to guarantee the topological invariance between an image and all of its transformed ones. This algorithm is based on $(i)$ the recently introduced notion of DRT graph [78, and (ii) the classical notion of simple point 910 . The first notion provides a way to exhaustively explore the space of transformed (sub)images while the second provides information on the possible topological modifications when performing such an exploration.

\section{Mathematical Tools for Topological Verification of Images under Rigid Transformations}

\subsection{Discrete Rigid Transformation Graph}

In opposition to rigid transformations in $\mathbb{R}^{2}$, discrete rigid transformations (DRTs) are not continuously defined w.r.t. the parameters $a, b$ and $\theta$. In particular, the parameter space $(a, b, \theta) \in \mathbb{R}^{3}$ is divided into $3 \mathrm{D}$ open cells, in each of which the functions $T_{a b \theta}$ are equal, while the $2 \mathrm{D}$ surfaces bounding these open cells represent to discontinuities of the DRTs, induced by the digitisation process (see Eq. (2)). In fact, each 2D surface is corresponded to an elementary modification of one pixel.

From a theoretical point of view, each $3 \mathrm{D}$ open cell can be seen as the equivalent class of the rigid transformations $\mathcal{T}$ that leads to a same DRT $T=D \circ \mathcal{T}$ whose boundaries are the 2D surfaces. By mapping any $3 \mathrm{D}$ cell onto a $0 \mathrm{D}$ point and any 2D surface onto a $1 \mathrm{D}$ edge, the combinatorial structure of the parameter space can be modeled in a dual way by a connected graph, as illustrated in Fig. 6. In particular, each 0D point corresponds to a transformed image generated by the associated $T$ and an $1 \mathrm{D}$ edge between two cells indicates that the two associated images differ at exactly one pixel.

Definition 2 (DRT graph [7]). A DRT graph $G=(V, E)$ is defined such that:

- any vertex $v \in V$ models a $3 D$ open cell and associates to a transformed image;

- any (labeled) edge $e=\left(v, w,\left(\boldsymbol{p}, \boldsymbol{p}^{\prime}\right)\right) \in E$ models a $2 D$ discontinuity surface between the transformed images corresponding to the DRTs $v$ and $w$ which differ at the single pixel $\boldsymbol{p}^{\prime}$, and $\boldsymbol{p}$ is the pixel corresponding to $\boldsymbol{p}^{\prime}$ in the original image. 

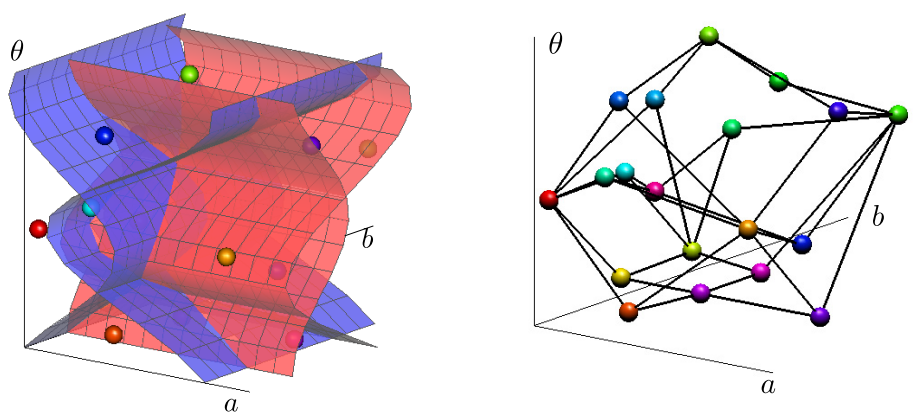

Fig. 6. A part of the parameter space subdivided by four $2 \mathrm{D}$ surfaces corresponding to the discontinuities of DRTs (left), and the associated part of the DRT graph (right)

The label $\left(\boldsymbol{p}, \boldsymbol{p}^{\prime}\right)$ on each edge is -implicitly- associated to a function indicating the value modification of the pixel $\boldsymbol{p}^{\prime}$ that differs between the transformed image associated to DRTs $v$ and $w$. More precisely, the value of $\boldsymbol{p}^{\prime}$ at the vertex $v$ is defined by $I_{v}\left(\boldsymbol{p}^{\prime}\right)=I(\boldsymbol{p})$ where $I: S \rightarrow F$ is the original image function. After the elementary rigid motion at $e$, we then obtain a new image $I_{w}$ by simply changing the pixel value at $\boldsymbol{p}^{\prime}$ as $I_{w}\left(\boldsymbol{p}^{\prime}\right)=I(\boldsymbol{p}+\boldsymbol{\delta})$ where $\boldsymbol{\delta}=( \pm 1,0)$ or $(0, \pm 1)$. Note that $\boldsymbol{\delta}$ corresponds to an elementary motion, i.e., a smallest pixel movement, that changes either $x$ - or $y$-coordinate by 1 .

It was proved in [7] that the DRT graph associated to a digital image of size $N^{2}$ has a space complexity of $\mathcal{O}\left(N^{9}\right)$ (and can be built with a similar time complexity [7/8]). Note that the structure of the DRT graph depends only on the support of the given images, but not on their pixel values. By construction, the DRT graph provides all the transformed images of a given image $I$. In particular, these transformed images can be generated by progressively and exhaustively scanning the DRT graph.

Remark 3. Let $I: S \rightarrow F$, and $G=(V, E)$ be its DRT graph. For each edge $e=\left(v, w,\left(\boldsymbol{p}, \boldsymbol{p}^{\prime}\right)\right)$ (i.e., each elementary modification of a pixel $\boldsymbol{p}^{\prime} \in S$ ), two cases can occur:

(i) $I_{v}\left(\boldsymbol{p}^{\prime}\right)=I_{w}\left(\boldsymbol{p}^{\prime}\right)$, i.e., the images of $I$ by the DRTs $v$ and $w$ are equal $\left(I_{v}=I_{w}\right)$;

(ii) $I_{v}\left(\boldsymbol{p}^{\prime}\right) \neq I_{w}\left(\boldsymbol{p}^{\prime}\right)$, i.e., $I_{v} \neq I_{w}$.

In the (considered) case of binary images, the value of $\boldsymbol{p}^{\prime}$ may then be flipped from white to black (or vice versa), and this may constitute the only modification between the transformed images of I by the DRTs $v$ and $w$.

\subsection{Topological Analysis of Binary Images from DRT Graphs}

From a DRT graph, one can generate exhaustively all the DRT images of an image $I$. Moreover, from Rem. 3] we know that this can be done by modifying (at most) one pixel value between two successive transformed images. In the case of binary images, it is actually possible to check if such a local modification involves a simple point. 


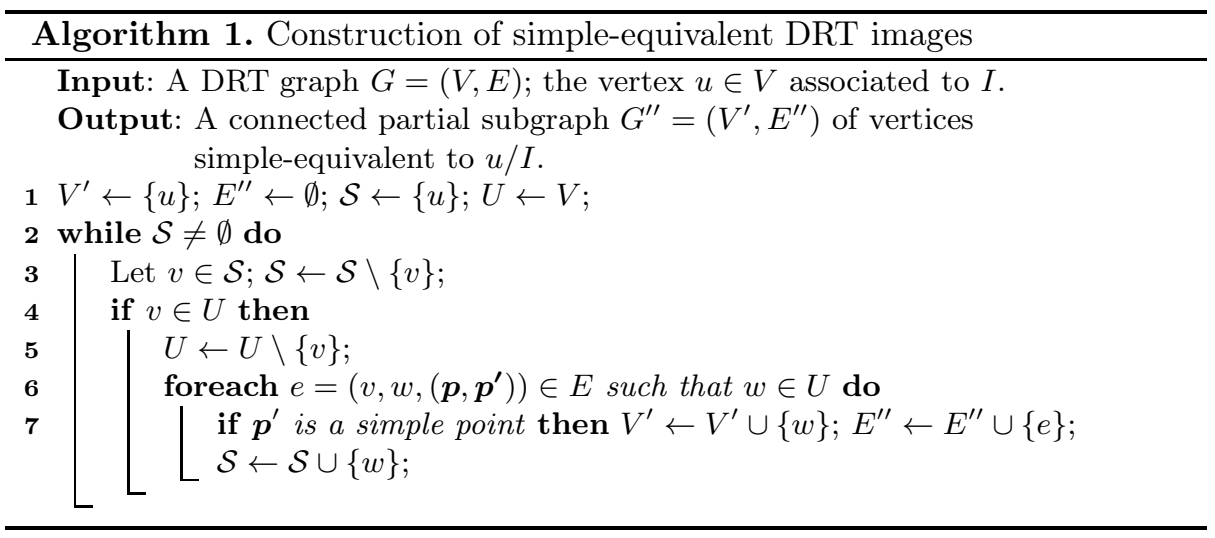

Practically, the edges of a DRT graph $G=(V, E)$ can then be classified in two categories: those that do not modify the topology of the transformed images (i.e., the edges that correspond to case $(i)$ in Rem. 3, as well as those that correspond to case ( $i i)$ for which $\boldsymbol{p}^{\prime}$ is a simple point); and those that modify this topology (i.e., the edges that correspond to case (ii) in Rem. 3 for which $\boldsymbol{p}^{\prime}$ is not simple).

The partial graph $G^{\prime}=\left(V, E^{\prime}\right)$ is obtained by maintaining only the edges $E^{\prime} \subseteq$ $E$ of the first category. $G^{\prime}$ is composed of connected components of vertices whose associated transformed images are simple-equivalent (see Sec.2.2), and thus have the same homotopy type. In particular, the connected component contains the vertex $u$ corresponding to the initial image $I$, as well as those corresponding to transformed images obtained from $I$ by elementary motion sequences which are topology-preserving. This specific set of vertices can be straightforwardly computed by using a standard spanning-tree algorithm, initialized from the seed vertex $u$ (see Alg. 1).

Remark 4. The connected component of $G^{\prime}$ that contains u may constitute only a strict subset of the vertices/transformed images that are simple-equivalent to $u / I$. Indeed, the edges of the DRT graph $G$ only model the local modifications associated to DRTs. In particular, there may exist other series of local modifications relying on simple points but not modeled in the DRT graph. In other words, the analysis of the DRT graph provides sufficient (but not necessary) conditions to guarantee homotopy-type preservation.

In the case where $V^{\prime}=V$ (see Alg. 1), i.e., when all the vertices of the DRT can be reached from $u$ by a sequence of edges involving only simple points, the algorithm successfully detects - as a side effect- an image $I$ that is actually topologically invariant under any DRTs. The algorithmic cost of this algorithm is directly linked to the size of the DRT graph, that is $\mathcal{O}\left(N^{9}\right)$. This algorithmic complexity is indeed reached in the worst cases. In the next section, we show that this problem can however be decomposed spatially, thus leading to a practical, lower complexity algorithm. 


\section{Local Evaluation of Topological Invariance under DRTs}

In the previous section, we have proposed to explore the whole DRT graph of an image $I$ in order to check its potential topological invariance under DRTs. For each edge $e=\left(v, w,\left(\boldsymbol{p}, \boldsymbol{p}^{\prime}\right)\right)$ of the DRT graph, we verify that the pixel $\boldsymbol{p}^{\prime}$ whose value is modified at this edge is actually a simple point for the transformed images $I_{v}$ and $I_{w}$. This test is performed locally, more precisely in the $3 \times 3$ neighbourhood centered on $\boldsymbol{p}^{\prime}$ in the transformed space.

We now take advantage of the local nature of these tests to develop a spatial decomposition strategy that will lead to a local version of the previously proposed global method. To this end, we first need to introduce basic notions and properties related to the influence of DRTs on pixel neighbourhoods.

\subsection{Neighbourhoods and DRTs}

Let $\boldsymbol{p} \in \mathbb{Z}^{2}$ be a pixel. We define the neighbourhoods of $\boldsymbol{p}$ as follows: $N_{8}(\boldsymbol{p})=$ $\left\{\boldsymbol{q} \in \mathbb{Z}^{2} \mid\|q-p\|_{2}<2\right\} ; N_{20}(\boldsymbol{p})=\left\{\boldsymbol{q} \in \mathbb{Z}^{2} \mid\|q-p\|_{2}<2 \sqrt{2}\right\}$. The first $3 \times 3$ neighbourhood is classically used in digital geometry and topology. The second corresponds to a $5 \times 5$ square from which the 4 extremal corner pixels have been removed. We provide the following property where we consider any arbitrary $\operatorname{DRT} T: \mathbb{Z}^{2} \rightarrow \mathbb{Z}^{2}$.

Property 5. Let $\boldsymbol{p} \in \mathbb{Z}^{2}$ and $\boldsymbol{q} \in N_{8}(\boldsymbol{p})$. We then have $T^{-1}(\boldsymbol{q}) \in N_{20}\left(T^{-1}(\boldsymbol{p})\right)$.

Proof. This property derives from the above definitions of the neighbourhoods, and from the fact that a DRT $T$ (due to the digitization induced by $D$, see Eq. (2) ) implies a possible (strict) majoration of $\sqrt{2}$ for the distance between transformed points, w.r.t. its associated rigid transformation $\mathcal{T}$.

\subsection{A Local Approach for Topological Analysis}

As stated above, a DRT graph models all the rigid transformations of a given digital image $I$. Despite the fact that the space of these transformations is actually infinite, the DRT graph is defined as a finite structure. This restriction can be made without loss of correctness/completeness by considering translation invariance. Indeed, a rigid transformation is defined as a composition of a rotation and a translation (Eq. (1) ). In particular, a rigid transformation $\mathcal{T}=\boldsymbol{t} \circ \boldsymbol{r}$, composed of a rotation $r$ around the origin and a translation $t \in \mathbb{R}^{2}$, can be also expressed as $\mathcal{T}=\boldsymbol{t} \circ \boldsymbol{t}^{\prime-1} \circ \boldsymbol{r}^{\prime} \circ \boldsymbol{t}^{\prime}$, where $\boldsymbol{t}^{\prime}$ is the translation by a vector $\boldsymbol{p} \in \mathbb{Z}^{2}$, and $\boldsymbol{r}^{\prime}$ is the rotation around $\boldsymbol{p}$. Such a translation in $\mathbb{Z}^{2}$ (that induces no topological modification, since the whole image is translated), allows any pixel $\boldsymbol{p}$ of $S$ of the image $I$ to be considered as the origin of $S$.

Let come back to the DRT graph $G=(V, E)$ considered until now, and involved in the global process defined in the previous section. We now focus on an edge $e=\left(v, w,\left(\boldsymbol{p}, \boldsymbol{p}^{\prime}\right)\right)$ of $G$. Obviously, the vertices $v$ and $w$ exist in $G$, and also in the (equivalent) DRT graph where we consider $\boldsymbol{p}$ as origin. In the later graph, any edge that does not involve in its label a pixel of $N_{20}(\boldsymbol{p})$ has no 
influence on the topological modifications in $N_{8}\left(\boldsymbol{p}^{\prime}\right)$ (see Prop. 5). Without loss of correctness, such topological modifications at $\boldsymbol{p}^{\prime}$ in $N_{8}\left(\boldsymbol{p}^{\prime}\right)$ (and thus of $\boldsymbol{p}^{\prime}$ in the whole image) only depends on the part of the DRT graph that corresponds to the restriction of $I$ to $N_{20}(\boldsymbol{p})$, denoted by $I_{\mid N_{20}(\boldsymbol{p})}$. Based on these considerations, it appears that if for any pixel $\boldsymbol{q}$ in the initial image $I$, the restriction $I_{\mid N_{20}(\boldsymbol{q})}$ does not lead to topological modifications under any DRTs, then the same conclusion holds for the whole image $I$. In other words, every elementary topological change occurring on the DRT graph $G$ of $I$ can be observed locally. Therefore, we need only to verify the topological invariance for every pixel of $I$ in its neighbourhood $N_{20}$ in the original binary image.

Proposition 6. Given a binary image $I: S \rightarrow F$, for every $\boldsymbol{p} \in S$ if $I_{\mid N_{20}(\boldsymbol{p})}$ is a local binary configuration which is topologically invariant under any rigid transformations, then the image $I$ is topologically invariant.

We assume that the modified pixel $\boldsymbol{p}^{\prime}$ at each elementary rigid modification of the DRT graph corresponds to the origin $\boldsymbol{o}_{\mathbf{2}}$ in the transformed image, and that the corresponding pixel $\boldsymbol{p}$ is the origin $\boldsymbol{o}_{\mathbf{1}}$ in the original image of size in its $N_{20}\left(\boldsymbol{o}_{\mathbf{1}}\right)$. Then, we simply need to construct the DRT graph with $\boldsymbol{p}^{\prime} \in[-1,1]^{2}$, $\boldsymbol{p} \in[-2,1]^{2}$ (i.e., the DRT graph of edges labels $\left(\boldsymbol{p}, \boldsymbol{p}^{\prime}\right)$ with this constraint), denoted by $G_{\boldsymbol{p}}=\left(V_{\boldsymbol{p}}, E_{\boldsymbol{p}}\right)$. We use Alg. 1 proposed in Sec.4.2 to verify in $G_{\boldsymbol{p}}$ the topological equivalence between two adjacent vertices $v$ and $w$ whose edge has the label $\left(\boldsymbol{o}_{\mathbf{1}}, \boldsymbol{o}_{\mathbf{2}}\right)$. If every edge has topologically equivalent vertices, then the center point $\boldsymbol{o}_{\mathbf{1}}$ of such a configuration is topologically invariant under any rigid transformations. This approach, in particular, leads to the following consideration: if we study the topological invariance property for all the binary image configurations of $N_{20}\left(\boldsymbol{o}_{\mathbf{1}}\right)$, we can identify a family of elementary configurations that authorise topological invariance under DRTs.

From Prop. 6, we propose a look-up-table-based algorithm for characterizing the topologically invariant property of any binary image. More precisely, we generate a set $P_{4}$ (resp. $\left.P_{8}\right)$ which contains only topologically invariant configurations in $(4,8)$ - (resp. $(8,4)$-) adjacent relations. Then we use $P_{4}$ and $P_{8}$ to verify whether the given image is topologically invariant. The method for building $P_{4}$ and $P_{8}$ is given in Alg. 2. Let $C$ be the set of all binary image configurations of size $N_{20}$, which is used to build $P_{4}$ and $P_{8},|C| \leq 2^{20}$. From Rem. 3 we have $I_{v} \neq I_{w}$ if $I_{v}\left(\boldsymbol{o}_{\mathbf{2}}\right) \neq I_{w}\left(\boldsymbol{o}_{\mathbf{2}}\right)$, where $I_{v}\left(\boldsymbol{o}_{\mathbf{2}}\right)=I\left(\boldsymbol{o}_{\mathbf{1}}\right)$ and $I_{w}\left(\boldsymbol{o}_{\mathbf{2}}\right)=I\left(\boldsymbol{o}_{\mathbf{1}}+\boldsymbol{\delta}\right)$. We thus need to consider the configurations of $N_{20}$ whose the central pixel value $I\left(\boldsymbol{o}_{\mathbf{1}}\right)$ and that of its 4-neighbouring pixel $I\left(\boldsymbol{o}_{\mathbf{1}}+\boldsymbol{\delta}\right)$ are different, e.g., $I\left(\boldsymbol{o}_{\mathbf{1}}\right)=1$ and $I\left(\boldsymbol{o}_{\mathbf{1}}+\boldsymbol{\delta}\right)=0$. Here we set $\boldsymbol{\delta}=(0,1)$, i.e., $\boldsymbol{o}_{\mathbf{1}}+(1,0)$ is the right pixel of $\boldsymbol{o}_{\mathbf{1}}$. In other words, the pixel values of $\boldsymbol{o}_{\mathbf{1}}$ and its right pixel $\boldsymbol{o}_{\mathbf{1}}+\boldsymbol{\delta}$ are pre-set. Under such conditions, $|C|$ is reduced to $2^{18}$. Thanks to the reflection and rotational symmetries, we can again reduce $|C|$ to 124260 . Then, we use Alg. 1 proposed in Sec. 4.2 to study the topologically invariant property of configurations in $C$. We store in $P_{4}$ and $P_{8}$ the subset of $C$ containing only the topologically invariant configurations w.r.t. the $(4,8)$ - and $(8,4)$-adjacent relations. Using Alg. 2, we obtain sets of 10643 and 19446 topologically invariant configurations in $P_{4}$ and $P_{8}$ respectively. Fig. 7 shows some elements of $P_{4}$ and $P_{8}$. 
Based on Prop. 6 and the sets $P_{4}$ and $P_{8}$, the algorithm for characterizing the topologically invariant property of a given binary image $I: S \rightarrow F$ by a local verification of pixels is given in Alg 3. The algorithm scans $I$ and considers for each pixel $\boldsymbol{p} \in S$ its $N_{20}(\boldsymbol{p})$ with either $P_{4}$ or $P_{8}$ depending on the binary value of $\boldsymbol{p}$. Note that $\bar{I}(\boldsymbol{p})=1-I(\boldsymbol{p})$.
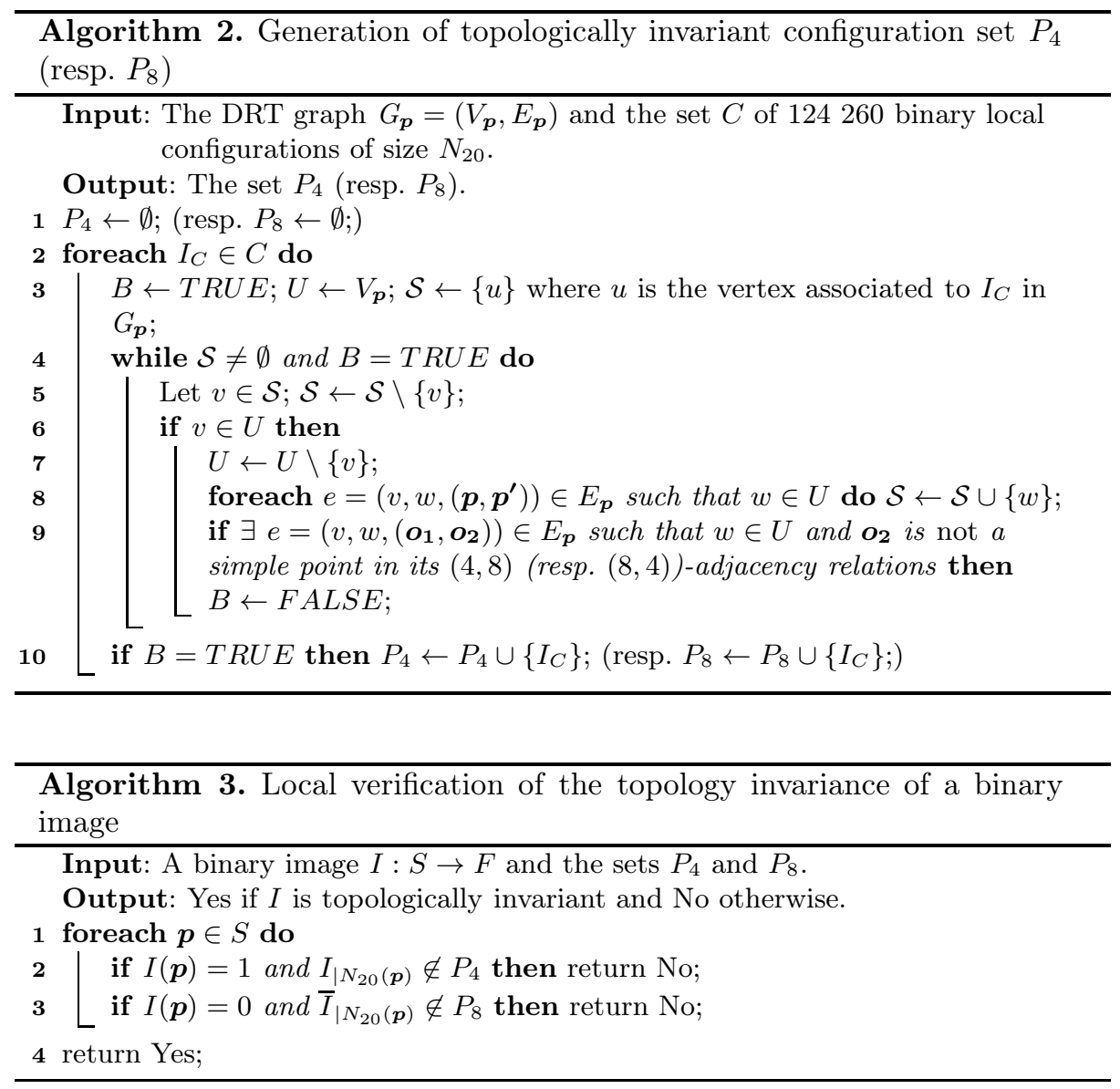

\section{Experiments}

In this section, we illustrate the relevance of our approach by presenting images which have been detected as topology-invariant (see Fig. [8(a-c), (d-f)), or topology-variant (see Fig. $8(\mathrm{~g}, \mathrm{~h}),(\mathrm{i}, \mathrm{j}),(\mathrm{k}, \mathrm{l}))$. Thanks to our LUT-based approach, such a detection can be carried out in linear time w.r.t. the image size.

As mentioned in Rem. 4, we only have a sufficient condition for homotopy-type preservation, so far we do not have a proof for a necessary condition. Nonetheless we have not found any example for which our algorithm fails to characterize its topological invariance. 


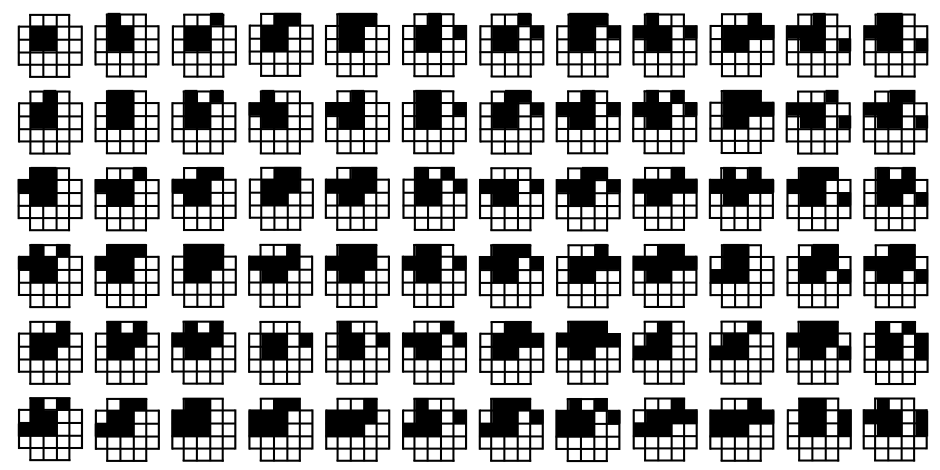

(a)

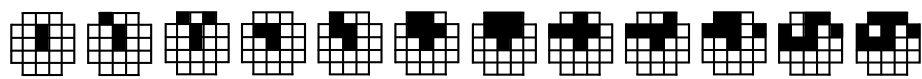

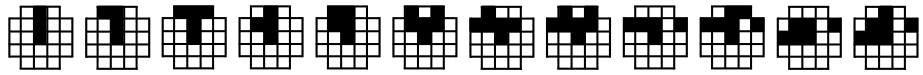
\%

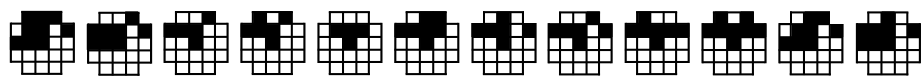

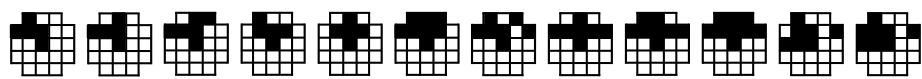

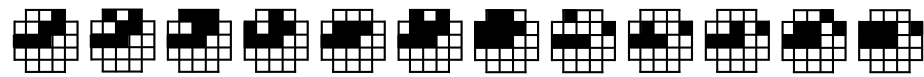

(b)

Fig. 7. Some topologically invariant configurations of $P_{4}(\mathrm{a})$ and $P_{8}$ (b)

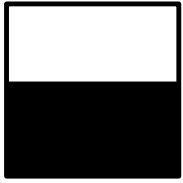

(a)

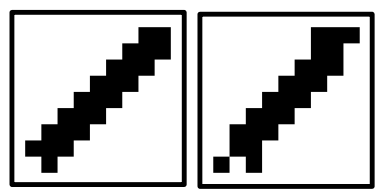

(g)

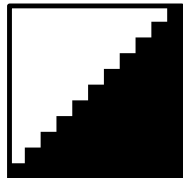

(b) (c)

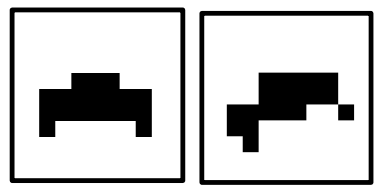

(h)

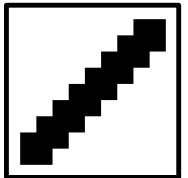

(d) (e)

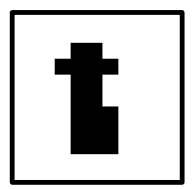

(i)

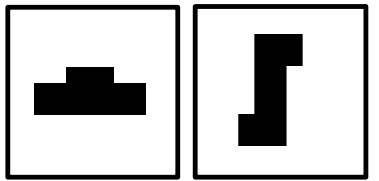

(f)

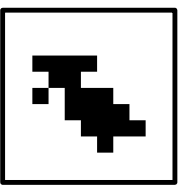

Fig. 8. (a-f) Some examples of topology-invariant images. (g-i) Three examples of topology-variant images (left) with their transformed images (right) exhibiting different topologies from their respective original images. 


\section{Conclusion}

We have proposed an algorithmic process for determining the topological invariance of digital images under any discrete rigid transformations. This work is based on the recently introduced notion of DRT graph [78, which presents a polynomial complexity that generally forbids its practical application on whole images. Nevertheless, DRT graphs have been successfully involved in a preliminary local analysis that finally led to a low complexity methodology, relying on image spatial decomposition.

Beyond its theoretical aspects, this work may contribute to the better understanding of the relationships that exist between geometry and topology in the framework of digital imaging, where both notions are more strongly linked than in continuous spaces.

This study was carried out in the context of binary images. However, it remains relevant whenever a notion of simple point (or more generally a local characterisation of topology preservation) is available. This is verified, for instance, in the context of $n$-ary images [14]. On the other hand, only the Eulerian (backwards) model has been considered in this study. In future work, we will extend these results to the case of the Lagrangian (forwards) model. Note that additional difficulties arise in the Lagrangian model, such as double pixels in the transformed space that may receive two different values, and null pixels that do not have any value. The Lagrangian model thus involves a value decision problem for such pixels.

\section{References}

1. Zitová, B., Flusser, J.: Image registration methods: A survey. Image and Vision Computing 21, 977-1000 (2003)

2. Yilmaz, A., Javed, O., Shah, M.: Object tracking: A survey. ACM Computing Surveys 38, 1-45 (2006)

3. Jacob, M.A., Andres, E.: On discrete rotations. In: DGCI, Proceedings, pp. 161174 (1995)

4. Andres, E.: The Quasi-Shear Rotation. In: Miguet, S., Ubéda, S., Montanvert, A. (eds.) DGCI 1996. LNCS, vol. 1176, pp. 307-314. Springer, Heidelberg (1996)

5. Nouvel, B., Rémila, E.: Configurations induced by discrete rotations: Periodicity and quasi-periodicity properties. DAM 147, 325-343 (2005)

6. Thibault, Y., Kenmochi, Y., Sugimoto, A.: Computing upper and lower bounds of rotation angles from digital images. Pattern Recognition 42, 1708-1717 (2009)

7. Ngo, P., Kenmochi, Y., Passat, N., Talbot, H.: Combinatorial structure of rigid transformations in 2D digital images. To appear in Computer Vision and Image Understanding

8. Ngo, P., Kenmochi, Y., Passat, N., Talbot, H.: Combinatorial Properties of 2D Discrete Rigid Transformations under Pixel-Invariance Constraints. In: Barneva, R.P., Brimkov, V.E., Aggarwal, J.K. (eds.) IWCIA 2012. LNCS, vol. 7655, pp. 234-248. Springer, Heidelberg (2012)

9. Kong, T.Y., Rosenfeld, A.: Digital topology: Introduction and survey. Computer Vision Graphics \& Image Processing 48, 357-393 (1989) 
10. Couprie, M., Bertrand, G.: New characterizations of simple points in 2D, 3D, and 4D discrete spaces. IEEE Transactions on Pattern Analysis and Machine Intelligence 31, 637-648 (2009)

11. Khalimsky, E.: Topological structures in computer science. Journal of Applied Mathematics and Simulation 1, 25-40 (1987)

12. Kovalevsky, V.A.: Finite topology as applied to image analysis. Computer Vision, Graphics \& Image Processing 46, 141-161 (1989)

13. Bertrand, G., Couprie, M., Passat, N.: A note on 3-D simple points and simpleequivalence. Information Processing Letters 109, 700-704 (2009)

14. Mazo, L., Passat, N., Couprie, M., Ronse, C.: Topology on digital label images. Journal of Mathematical Imaging and Vision 44, 254-281 (2012) 